Sociologie et sociétés

\title{
Les disparités en matière de santé au fil du vieillissement
}

Une comparaison du parcours de vie des premiers

baby-boomers et des pré--baby-boomers au Canada

\section{Health Disparities as We Age}

\author{
A Life Course Comparison of Canadian Early Boomers with
}

Pre-Boomers

\section{Susan A. McDaniel, Amber Gazso, Hugh McCague et Ryan Barnhart}

Volume 45, numéro 1, printemps 2013

Inégalités, parcours de vie et politiques publiques

Inequality, Life Course and Public Policy

URI : https://id.erudit.org/iderudit/1016395ar

DOI : https://doi.org/10.7202/1016395ar

Aller au sommaire du numéro

Éditeur(s)

Les Presses de l’Université de Montréal

ISSN

0038-030X (imprimé)

1492-1375 (numérique)

Découvrir la revue

Citer cet article

McDaniel, S. A., Gazso, A., McCague, H. \& Barnhart, R. (2013). Les disparités en matière de santé au fil du vieillissement : une comparaison du parcours de vie des premiers baby-boomers et des pré-baby-boomers au Canada. Sociologie et sociétés, 45(1), 43-65. https://doi.org/10.7202/1016395ar 


\title{
Les disparités en matière de santé au fil du vieillissement
}

\author{
Une comparaison du parcours de vie des premiers \\ baby-boomers et des pré-baby-boomers au Canada
}

\section{SUSAN A. MCDANIEL}

Prentice Institute for Global Population and Economy

Lethbridge University

Lethbridge (Alberta) $\mathrm{T}_{1} \mathrm{~K}_{3} \mathrm{M}_{4}$

Courriel: susan.mcdaniel@uleth.ca

\section{AMBER GAZSO}

Department of Sociology

2060, Vari Hall

York University

Toronto (Ontario) MKN 3 M6

Courriel: agazso@yorku.ca

\author{
HUGH MCCAGUE \\ Institute for Social Research \\ York University \\ 4700 Keele Street \\ Toronto (Ontario) $\mathrm{M}_{3} \mathrm{I}_{1} \mathrm{P}_{3}$ \\ Courriel: hmccague@yorku.ca
}

\author{
RYA N BAR N HART \\ Department of Political Science \\ Ross Building \\ York University \\ Toronto (Ontario) MKN $3 \mathrm{M} 6$ \\ Courriel: rbarnhart@yorku.ca
}

Traduction: Isabelle Malo

$\mathrm{L}$ A SANTÉ ET LE BIEN-ÊTRE FUTURS des baby-boomers représentent un intérêt important et croissant dans la recherche ${ }^{1}$ et la politique, car les membres du premier échelon de cette cohorte ont atteint l'âge de 65 ans en $2011^{2}$. Étonnamment, rares

1. Remerciements: La recherche est financée par le Conseil de recherches en sciences humaines du Canada. (CRSH) Nous remercions les centres de données de recherche de l'Université York et de l'Université de Lethbridge pour leur aide et leurs conseils sur l'accès aux données. Le présent article est une version largement révisée d'un document présenté à la Conférence socio-économique de Statistique Canada, qui a eu lieu à Gatineau, les 2 et 3 mai 2011. Nous tenons également à remercier un certain nombre de collègues pour leurs commentaires utiles, en particulier Lisa Strohschein et Michael Wolfson. Ajoutons que les auteurs sont responsables de toute erreur et que Paul Bernard était codemandeur pour ce projet.

2. Les plus jeunes membres de la cohorte n'atteindront l'âge de 65 ans qu'en 2031 . 
sont les recherches qui ont été effectuées à partir d'une perspective fondée sur les parcours de vie et axée sur le vieillissement, lors du passage du milieu vers la fin de la vie adulte ${ }^{3}$. Comme le suggèrent Seabrook et Avison (2012:63), l'adoption d'une approche prospective est particulièrement nécessaire en période de difficultés et de transition socio-économiques. L'absence d'une telle approche est d'autant plus surprenante compte tenu des discours récurrents, politiques et publics, sur la «crise» du vieillissement de la population. En politique ou dans les médias, on présume souvent simplement que l'importante génération des baby-boomers au Canada, née entre 1946 et 1966, aura une trajectoire de santé semblable à celle des générations plus âgées actuellement et qu'elle sera confrontée à des enjeux similaires. Pourtant, nous savons qu'au cours des prochaines décennies, le vieillissement pourrait être très différent de ce qu'il est aujourd'hui (voir par exemple Park, 2011) en ce qui a trait à l'évolution des modèles de travail, de retraite et de santé, et à l'évolution des familles de Canadiens qui vieillissent (Ménard, Le Bourdais et Hamplova, 2010). Wister (2005) constate un paradoxe sur la santé associé à l'imposante génération des baby-boomers à mesure qu'elle vieillit: à certains égards, les baby-boomers sont plus en santé que les membres des générations précédentes, mais ils sont plus susceptibles d'avoir un excès de poids et d'en subir les risques pour la santé. D'autres recherches suggèrent que le fait d'évoluer dans un monde marqué par la dissolution des mariages et des unions libres (Avison et al., 2007) ainsi que par des risques accrus de pauvreté (Turner, Wheaton et Lloyd, 1995) peut avoir des répercussions, tant physiques que psychologiques, sur les baby-boomers à mesure qu'ils prennent de l'âge. Par ailleurs, de nouvelles recherches (McDaniel, Gazso et Um, 2013) ont établi que les personnes ayant atteint la force de l'âge durant la période de récession qui a débuté en 2008 ont vu leurs perspectives financières compromises pour la dernière partie de leur vie, tout en étant confrontées à la nécessité accrue de soutenir des membres de la famille plus jeunes et plus âgés.

La façon dont les disparités en matière de santé évoluent tout au long de l'existence et en particulier du milieu à la fin de la vie n'est pas bien comprise en dépit de la recherche importante et croissante sur la santé en fonction du statut socio-économique (SSE) (Hertzman et Siddiqi, 2009; McDaniel, 2011; Seabrook et Avison, 2012; Singh-Mancoux et al., 2004). Une partie de la difficulté réside dans la complexité du lien bidirectionnel du SSE avec la santé ou le bien-être. En effet, il est reconnu que le SSE influence la santé et le bien-être d'une façon compliquée et en fonction du temps (House, 2002; Sacker et al., 2007; Seabrook et Avison, 2012). Toutefois, la santé et le bien-être ont aussi une influence sur le SSE: les personnes en mauvaise santé sont moins susceptibles d'être productives ou de connaître le succès économique. Lorsqu'il est question des personnes dans la force de l'âge, la complexité augmente pour au moins deux raisons. Tout d'abord, ces personnes ont cumulé au cours de leur vie des avantages ou des inconvénients qui peuvent avoir une incidence sur leur bien-être. Ce

3. Une exception notable est celle de Singh-Manoux et al. (2004) qui s'appuie sur les données de Whitehall. 
cumul peut cependant être «en dents de scie», par exemple, lorsque des crises économiques surviennent et que les avantages cumulés diminuent. De plus, les personnes qui ont atteint la mi-vie sont habituellement en interrelation avec des membres de la famille plus âgés et plus jeunes touchés par les mauvaises expériences des autres générations. Par conséquent, même si les personnes dans la force de l'âge peuvent être moins touchées par le ralentissement économique, par comparaison avec des plus jeunes par exemple, elles peuvent être obligées de soutenir ces derniers, peut-être même au détriment de leur propre sécurité d'avenir.

Dans le présent article, nous comparerons deux cohortes de la génération des baby-boomers, les premiers baby-boomers nés entre 1947 et 1951 et les pré-babyboomers nés entre 1932 et 1936, sur une période de quatorze à quinze ans (1994-1995 à 2008-2009) durant laquelle les inégalités du revenu se sont accrues (voir Heisz, 2007; Picot et Myles, 2005).

Pour ce faire, nous avons établi les quatre questions principales suivantes:

- De quelle façon les disparités en matière de santé en fonction du SSE influencentelles le passage du milieu à la fin de la vie chez les deux cohortes de Canadiens durant la période de 1994-1995 à 2008-2009?

- Comment la santé des premiers baby-boomers peut-elle être comparée avec celle des pré-baby-boomers en période d'accroissement des inégalités du revenu?

- Les disparités en matière de santé s'accentuent-elles pour ces deux cohortes?

- Le vieillissement accru de la population canadienne aura-t-il des conséquences pour l'avenir?

\section{CONTEXTE}

Nous savons depuis longtemps que le SSE est lié à la santé ou au bien-être de nombreuses façons. Il s'agit de l'une des variables explicatives les plus fiables concernant les disparités en matière de santé (Hertzman et Siddiqi, 2009; Ross et al., 2011; Seabrook et Avison, 2012). Une recherche en plein essor permet de documenter les disparités en matière de santé en fonction du SSE (à titre d'exemple, Black Report, 1980; House, 2002; Ross et al., 2012; Willson, 2009). Au Canada, on estime que $25 \%$ des années de vie perdues prématurément sont attribuables à l'inégalité du revenu (Wilkins et al., 2002). Pourtant, peu d'études ont été réalisées sur les adultes dans la force de l'âge ou plus âgés (c.-à-d. des adultes âgés de 45 à 64 ans) qui permettent d'observer l'évolution de personnes entre le milieu et la dernière partie de leur vie. L'étude Whitehall (Black Report, 1980) est une exception notable, qui a permis de constater que la hiérarchie sociale a une influence très importante sur la santé, même en excluant un contexte de pauvreté ou de privation importante. Sacker et al. (2007: 812) expliquent la nécessité d'une recherche longitudinale: «[...] la plupart [...] des recherches s'appuient sur des données transversales, même s'il est généralement admis que les conditions socio-économiques et la santé ont une relation complexe et dépendante du temps et que l'analyse de cette relation nécessite des données longitudinales 
de mesures répétées.» De leur côté, Singh-Mancoux et ses collègues (2004: 1073) avancent que puisque le SSE d'un adulte résume de façon très efficace les trajectoires sociales de son parcours de vie, il est important d'examiner les avantages et les inconvénients cumulés au cours de la vie adulte.

Nous proposons de faire un examen comparatif des parcours de vie de personnes qui font partie de deux cohortes et dont les antécédents sont marqués par l'accroissement des inégalités du revenu au Canada à différents moments historiques et biographiques. Un aperçu des expériences de vie de nos deux cohortes analysées est présenté dans le tableau 1. Les pré-baby-boomers constituent une petite cohorte née à la suite de la grande dépression, entre 1932 et 1936. Ils ont atteint l'âge adulte au milieu des années 1950, dans la période de croissance économique fulgurante qui a suivi la Seconde Guerre mondiale. En effet, il s'agissait d'une époque où un revenu salarial pouvait faire vivre une famille entière, où le taux de chômage était faible et le logement bon marché, et où l'on a assisté à l'émergence de l'État-providence canadien. Cette cohorte est entrée à mi-vie à la fin des années 1970 ou au début des années 1980, alors que les inégalités du revenu s'accentuaient, mais dont les effets ont été «entièrement compensés par les systèmes fiscaux et les systèmes de transferts » (Frenette, Green et Milligan, 2006: 26). Lorsque nous avons commencé à les suivre, les membres de cette cohorte étaient âgés de 58 à 62 ans en 1994-1995.

Les premiers baby-boomers, nés entre 1947 et 1951, font en revanche partie d'une vaste cohorte née peu de temps après la fin de la Seconde Guerre mondiale. Ils ont atteint l'âge adulte entre 1969 et 1973, alors que l'économie prenait un certain retard, exacerbé par le fait que ces baby-boomers tentaient d'obtenir un diplôme d'études postsecondaires et un emploi dans un marché très compétitif. Ils ont atteint la force de l'âge, c'est-à-dire, de 45 à 49 ans, entre 1992 et 2000, période au cours de laquelle nous avons commencé à les suivre. Il s'agit également d'une période où les inégalités du revenu au Canada ont enregistré une hausse et n'ont pas été tout à fait compensées de la même façon que dans les années 1980, par des transferts et des programmes de sécurité sociale (Frenette, Green et Milligan, 2006: 26). En outre, au début des années 2000, la proportion de $1 \%$ des déclarants de revenus les plus élevés a augmenté à $11 \%$ (contre $7 \%$ dans les années 1980 et $8 \%$ dans les années 1990) (Statistique Canada, 2013).

Deux théories établissent le lien du SSE avec le bien-être. La première est la théorie de la cause fondamentale (voir Willson, 2009) qui soutient que le SSE influence la façon dont nous expérimentons les risques pour la santé, alors même que les facteurs de risque se transforment. Les personnes qui ont un SSE élevé maitrisent davantage les ressources et le capital social, notamment les connaissances et l'accès à l'information en matière de santé, et sont mieux outillées pour éviter les menaces à la santé. Cette théorie avance également que les disparités en matière de santé en fonction du SSE persistent parce que les personnes d'un SSE élevé sont davantage en mesure d'agir lorsque de nouvelles preuves se dégagent (par exemple, les risques du tabagisme ou 
Tableau 1: Les cohortes de l'étude selon les âges clés, les dates et contextes importants en fonction de chaque période de temps examinée (la zone grise correspond à la période de notre étude longitudinale).

\begin{tabular}{|c|c|c|c|c|}
\hline Âge & Pré-baby-boomers & Premier & pomers & Contextes \\
\hline Années de naissance & $1932-1936$ & $1947-1951$ & \multicolumn{2}{|c|}{$\begin{array}{l}\text { Petite cohorte, années suivant la grande dépression } \\
\text { Grande cohorte, années suivant la Seconde Guerre mondiale }\end{array}$} \\
\hline Âge: 22 ans (entrée dans l'âge adulte) & $1954-1958$ & $1969-1973$ & \multicolumn{2}{|c|}{$\begin{array}{l}\text { Essor économique: salaire suffisant pour soutenir une famille entière, faibles } \\
\text { taux de chômage } \\
\text { Retard économique, marché compétitif avantageux pour l'éducation et l'emploi }\end{array}$} \\
\hline Âge : $45-49$ ans (mi-vie) & $1977-1985$ & $1992-2000$ & \multicolumn{2}{|c|}{$\begin{array}{l}\text { Inégalités du revenu en croissance, mais compensées par des transferts sociaux } \\
\text { 1994-1995 Peu de transferts/inégalités croissantes }\end{array}$} \\
\hline Âge: $59-64$ ans (fin de la mi-vie) & $1991-2000$ & $2006-2011$ & \multirow[b]{2}{*}{ 2008-2009 } & $\begin{array}{l}\text { Croissance importante du } 1 \% \text { des revenus les plus élevés } \\
\text { Ralentissement économique à partir de } 2008\end{array}$ \\
\hline Âge 70-74 ans (fin de vie) & $2006-2010$ & $2017-2025$ & & $\begin{array}{l}\text { Le ralentissement économique et d'importantes inégalités du } \\
\text { revenu peuvent avoir des effets négatifs sur les deux cohortes } \\
\text { analysées }\end{array}$ \\
\hline
\end{tabular}


d'une mauvaise alimentation). Il s'agit d'un point important sur le plan sociologique et politique, car le SSE continuerait d'être un déterminant essentiel de la santé et du bien-être, même si divers médiateurs entre le SSE et la santé étaient diminués. C’est ainsi que les différences socio-économiques en matière de santé persistent même avec des systèmes de soins de santé comme au Canada où tous ont un accès égal à des soins médicaux, peu importe le revenu (Prus, 2011; Wilkins, Berthelot et Ng, 2002).

Willson appuie la théorie de la cause fondamentale à partir d'une analyse longitudinale des trajectoires du SSE et de la santé, au Canada et aux États-Unis. Par exemple, des antécédents de faibles revenus augmentent les risques d'être atteint d'une maladie évitable aux États-Unis, mais pas au Canada, ce qui laisse supposer que le taux supérieur d'inégalités aux États-Unis peut être un facteur d'influence aussi important que les différentes politiques sociales des deux pays, en particulier, le système public d'assurance-maladie universel au Canada. Les recherches de Willson révèlent que même si les effets du SSE et des inégalités dans les sociétés ont une grande influence sur la santé, ils ne sont pas immuables et peuvent être compensés par de bonnes politiques et par l'aplanissement de la répartition des inégalités du revenu.

La deuxième théorie avance que les données sur la santé de la population et le gradient social permettent d'affirmer que plus les inégalités se creusent, plus la santé est menacée dans l'ensemble de la population, particulièrement chez les groupes qui ont un faible SSE (Wilkinson, 2006; Wilkinson et Pickett, 2006). Les disparités s'accentuent donc à mesure que les inégalités augmentent. De plus, chaque gradient social est caractérisé par une moins bonne santé que le gradient qui lui est supérieur, ce qui confirme les conclusions de l'étude Whitehall (Black Report, 1980). Cette constatation permet de compléter et d'élaborer la théorie de la cause fondamentale en confirmant non seulement la persistance des disparités en matière de santé en fonction du SSE, mais la façon dont celles-ci s'organisent par rapport à un continuum et à des changements à mesure qu'augmentent les inégalités.

Les quelques études empiriques longitudinales montrent que les gradients sociaux les plus élevés en matière de santé ont été relevés aux États-Unis (McDonough, Worts et Sacker, 2009). Un désavantage observé dans quatre pays de l'OCDE (GrandeBretagne, Danemark, Allemagne et États-Unis) a eu un effet important sur les trajectoires de santé avec le vieillissement. En revanche, il y a toutefois moins de différences dans les trajectoires de ceux qui sont avantagés, c'est-à-dire qui ont un SSE élevé, ce qui tend à confirmer l'idée que le désavantage n'est pas l'inverse de l'avantage dans une perspective fondée sur le parcours de vie en matière de santé (Ferraro et Shippee, 2009; McDaniel et Bernard, 2011).

La présente étude a pour cadre une perspective fondée sur le parcours de vie (McDaniel et Bernard, 2011), à partir des quatre principes directeurs suivant: 1) les expériences quotidiennes constituent une trame qui commence à la naissance et se poursuit jusqu'à la mort ; 2) les parcours de vie se déroulent selon de multiples dimensions interconnectées; 3 ) des liens sociaux se créent tout au long de notre parcours de vie, avec les autres et les institutions qui nous influencent; et 4) divers contextes géo- 
graphiques et nationaux façonnent les parcours de vie et sont à leur tour façonnés par eux. À l'aide du premier et du quatrième principe, nous examinons comment l'état de santé autodéclaré (ESAD) évolue, du milieu à la fin de l'existence, et la façon dont les contextes, en particulier le SSE et les inégalités, influencent cette évolution selon l'époque où naît une personne.

L'ESAD est l'état de santé d'un déclarant tel qu'il le perçoit lui-même et a été désigné comme un indicateur utile de la santé et du bien-être global des personnes et des populations. En tant qu'outil de mesure fiable et valide (Idler et Benyamini, 1997; Prus, 2011) pour l'évaluation de l'état de santé général (Idler, Russell et Davis, 1992), l'ESAD est aussi considéré comme une variable explicative efficace de la mortalité (Mossey et Shapiro, 1982; van Doorslaer et Gerdtham, 2003) et de l'invalidité (Mansson et Rastam, 2001), mais aussi de la limitation fonctionnelle (Idler et Benyamini, 1997), des comportements liés à la santé (Cott et al., 1997), et du recours aux soins de santé (Pinquart, 2001). Banks et al. (2006) ont constaté que les mesures de l'ESAD sont presque identiques aux mesures obtenues de façon biologique. Toute mesure comporte bien sûr des limites. En effet, toute mesure subjective de la santé peut être influencée par les différents seuils de tolérance entre des personnes ou des groupes. La signification et la présentation de l'ESAD peuvent aussi varier entre les groupes.

Notre intérêt pour l'ESAD, un indicateur de santé bien établi, provient de preuves empiriques qui laissent supposer que l'ESAD évolue durant le parcours de vie. Par exemple, McDonough, Worts et Sacker (2009) ont établi que l'ESAD en Allemagne et aux États-Unis reste relativement stable chez les jeunes adultes, qu'il décline à mi-vie, et qu'il redevient plus stable plus tard dans l'existence. Toutefois, ils ont observé un déclin constant dans l'ESAD en Grande-Bretagne et au Danemark au cours de la vie active. Notre objectif est d'examiner les trajectoires au Canada, du milieu à la fin de la vie dans les deux cohortes analysées.

\section{MÉTHODES ET DONNÉES}

Les données analysées proviennent de l'Enquête nationale sur la santé de la population (ENSP) du Canada, sur une période couvrant huit cycles (1994-1995 à 2008-2009). Nous mettons surtout l'accent sur la comparaison de deux cohortes sélectionnées, décrites précédemment (voir le tableau 1): la cohorte des premiers baby-boomers née entre 1947 et 1951 et la cohorte des pré-baby-boomers née entre 1932 et 1936. Les premiers baby-boomers sont âgés de 43 à 47 ans au premier cycle de l'ENSP (19941995 ) et de 59 à 64 ans au second cycle examiné (2008-2009) tandis que les pré-babyboomers sont âgés de 58 à 62 ans au premier cycle et de 74 à 79 au second. Les 17276 répondants de départ ont été divisés au hasard en deux ensembles de données: un ensemble de données exploratoires et un ensemble de données de confirmation. Un modèle de régression à effets contrastés et à plusieurs niveaux a été réalisé en utilisant toutes les données disponibles. Les modèles finaux testés à partir de l'ensemble de données de confirmation ont été limités aux personnes âgées de 16 à 95 ans afin 
d'éviter les données instables ou périphériques, ce qui contribue à renforcer la fiabilité des résultats globaux (voir Fox, 2008).

Notre variable dépendante est l'état de santé autodéclaré (ESAD), une mesure standard jugée constante pour tous les cycles de l'ENSP et utilisée avec une grande fiabilité et validité dans de nombreuses études (par exemple, Maio et Kemp, 2010; Sacker et al., 2007; White et al., 2011) ainsi que dans les recherches citées plus haut. Les répondants doivent évaluer leur état de santé par rapport à celui d'autres personnes de leur âge selon une échelle de cinq $(0=$ faible, $1=$ moyen, $2=$ bon, $3=$ très bon et 4 = excellent). À partir de l'état de santé le plus souvent désigné comme «très bonne santé», les données indiquent un certain nombre de biais. Dans la distribution marginale, ces biais ne posent toutefois pas de problème majeur pour les estimations de probabilités maximales (Gelman et Hill, 2007) et n'ont donc pas été modifiés.

Les covariables et les variables explicatives du modèle ont été tirées des données disponibles de l'ENSP et d'autres sources de Statistique Canada, notamment des variables socio-économiques comme le niveau de scolarisation, la situation d'activité, le revenu relatif, le sexe et les mesures de l'inégalité du revenu. Le niveau de scolarisation est mesuré à partir du plus haut niveau de scolarisation atteint. La variable comporte quatorze niveaux allant de «aucune instruction» à «doctorat obtenu». Cette variable a démontré une certaine variation dans le temps avec l'augmentation du niveau de scolarisation dans certains cas au cours des huit cycles. Le niveau de scolarisation a également été considéré comme variant dans le temps dans notre analyse. L'effet d'être au chômage ou de ne pas faire partie de la population active a été jugé comme variant dans le temps, avec la catégorie de référence utilisée ou adéquate. Les variables d'interaction basées sur le temps ont également été créées pour évaluer les effets combinés possibles de ces conditions au fil du temps et pour évaluer les différences dans l'effet du chômage pouvant être associé à un moment précis de la vie où une personne s'est trouvée sans emploi. Nous avons intégré le revenu du ménage à notre modèle en utilisant le ratio du revenu du ménage de l'ENSP qui tient compte à la fois de la taille des familles et de la résidence en milieu urbain ou rural. Ces données proviennent des calculs du seuil de faible revenu (SFR) de Statistique Canada, le niveau auquel les dépenses financières majeures d'une famille sont consacrées aux besoins essentiels. En deçà de ce niveau, les familles sont catégorisées comme ayant un faible revenu. Le revenu total du ménage est ensuite évalué en fonction de SFR particuliers afin d'établir un ratio pour chaque ménage qui devient alors une variable à distribution continue plutôt qu'une variable catégorique. Dans nos modèles, nous considérons cette variable comme en fonction du temps.

Un code fictif a été attribué au sexe, les hommes servant de catégorie de référence. Le sexe selon l'âge, le temps et les interactions dans le temps ont également reçu un code servant à évaluer les différences de trajectoires possibles entre les hommes et les femmes durant le parcours de vie. L'interaction à trois critères (femmes, selon l'âge, selon le temps) permet d'obtenir des connaissances analytiques plus approfondies sur les trajectoires de la dernière partie de la vie en tenant compte des différences poten- 
tielles entre les femmes et les hommes sur de longues périodes de temps et plus particulièrement au cours de la dernière partie de la vie. Afin d'évaluer les avantages possibles d'une relation de couple sur la santé, les personnes qui ont déclaré être mariées ou cohabiter avec leur partenaire ont reçu un code fictif par rapport à un groupe de référence dont les membres ont déclaré vivre dans un autre type de ménage. Cette variable a été considérée en fonction du temps, car évidemment des preuves d'évolution du mode de vie durant les huit cycles de l'ENSP ont été réunies.

Dans notre analyse, l'âge et les effets de l'âge influencent à la fois les personnes et les populations et nous avons recours à trois mesures liées à l'âge. Lorsqu'elles sont utilisées ensemble, ces mesures ont des propriétés linéaires et non linéaires qui permettent d'atteindre le bon ajustement empirique par rapport aux données observées au cours de l'existence. Les variables de l'âge et de l'âge moyen sont utilisées sur le plan individuel. L'âge moyen est une variable contextuelle (Monette, 2010; Snijders et Bosker, 2012) qui donne à chaque répondant une estimation de son état de santé par rapport à ses pairs du même âge. Cette variable permet d'estimer les effets sur la santé variables en fonction de l'âge, par rapport aux effets sur la santé sur le plan individuel. Afin d'examiner la complexité des relations entre ces variables et nos variables dépendantes, des conditions d'interaction en fonction du temps ont été établies. L'âge selon l'interaction en fonction du temps est une variable utilisée à l'échelle de la population qui permet de faire des comparaisons entre les deux cohortes analysées qui nous intéressent, celle des premiers baby-boomers et celle des pré-baby-boomers. L'effet de l'âge en fonction du temps a été intégré dans les modèles, car cette variable ouvre l'accès directement à des questions à l'échelle individuelle ou de la population, par exemple celle de savoir si la cohorte des baby-boomers et celle des pré-baby-boomers vivent et évaluent leurs problèmes de santé de façon semblable. Plus précisément, cette variable permet de se demander si une personne d'un âge donné par rapport à une autre personne du même âge, dont la seule différence réside dans l'endroit où elle se situe dans le temps (les deux cohortes analysées), doit s'attendre à avoir une trajectoire différente en matière de santé. L'interaction de l'âge en fonction du temps se comporte aussi comme une variable non linéaire, ce qui ouvre la voie à l'analyse de changements possibles liés à l'âge aux différents cycles de l'ENSP.

Le temps a été remis à zéro au premier cycle étudié de l'ENSP à son point milieu, entre les points de données de 1994 et de 1995. La même stratégie du point milieu est utilisée pour chaque cycle correspondant, ce qui entraîne une augmentation d'une unité dans le temps, une modification d'intervalle de deux ans environ. Cette détermination du temps vise à fournir une association de temps cohérente aux coefficients de Gini par année, obtenus auprès de Statistique Canada (s.d.). Le coefficient de Gini est déclaré sur une base annuelle et est rétrospectif.

Nous utilisons le coefficient de Gini comme mesure de l'inégalité du revenu. Ce coefficient varie entre 0 et $1:$ s'il est égal à 0 , le revenu ou le patrimoine doit être divisé sur une base complètement égale ou égalitaire, s'il est près de 1 , le revenu ou le patrimoine se rapproche davantage du sommet de la répartition. Les coefficients de Gini 
antérieurs ont été obtenus par les archives en ligne de Statistique Canada (Statistique Canada, s.d.). Le coefficient utilisé dans notre cas est le coefficient de Gini national pour mesurer le revenu total du ménage, en tenant compte de toutes les unités familiales, de 1992 à 2009. Les valeurs ont ensuite été associées au point milieu de chaque année de chaque cycle de l'ENSP, en prenant la valeur moyenne du coefficient de Gini pour chaque année de l'ENSP. Ainsi, pour le cycle 1, le coefficient de Gini utilisé représente la moyenne du coefficient de Gini pour 1994 et 1995. Par conséquent, nous obtenons une valeur plus représentative que les résultats atteints à la fin de certaines années en particulier. Afin d'étudier les effets combinés de l'inégalité du revenu en fonction du temps, une interaction entre une variable de Gini et le temps a été créée et intégrée dans nos modèles, ce qui donne un effet sur la trajectoire du coefficient de Gini.

Un modèle de régression linéaire à effets contrastés et à plusieurs niveaux a été réalisé en utilisant toutes les données disponibles. Ce modèle a été élaboré de façon longitudinale à l'aide de mesures particulières intégrées au niveau individuel, à partir duquel elles ont été prises. Des estimations ont été effectuées pour des interceptions aléatoires sur le plan individuel ainsi que pour un coefficient de pente aléatoire en incluant un effet aléatoire de temps dans le modèle au niveau 2. Cette structure hiérarchique permet d'établir le modèle de niveau 1 suivant:

$$
Y_{i j}={ }_{0 j}+{ }_{1 j} X_{i j}+\pi Z_{i}+R_{i j}
$$

La valeur $Y_{i j}$ représente les mesures individuelles prises à partir d'une personne $j$ à un moment $i ; Y_{0 j}$ est l'interception individuelle particulière pour une personne $j$; représente le vecteur colonne $1 \mathrm{x} n$ des coefficients de régression pour le vecteur $n \times 1$ des variables explicatives $Z_{i}$, pour une personne $j$ à un moment $i$. La variable $R_{i j}$ représente les résidus du niveau 1 répartis de façon indépendante et normale pour une personne $j$ à un moment $i$. Les interceptions individuelles de régression spécifiques et les coefficients de pente aléatoire au niveau 2 peuvent être exprimés de la façon suivante:

$$
\begin{gathered}
{ }_{1 j}={ }_{0}+U_{0 j} \\
{ }_{1 j} X_{1 j}={ }_{1}+U_{1}
\end{gathered}
$$

Dans ce cas, $\beta_{0} \beta_{0}$ correspond à l'interception (interception moyenne) des moindres carrés ordinaires (MCO) et $\beta_{1}$ à la pente (pente moyenne) des MCO pour toutes les personnes. Les valeurs $U_{0 j}$ représentent les écarts aléatoires estimés à partir de l'interception moyenne pour une personne $j$, et les valeurs $U_{1 j}$ peuvent être interprétées comme l'interaction aléatoire entre une personne $j$ à un moment $i$.

\section{Données manquantes, poids des données et validation du modèle}

Dans le but d'éviter les biais potentiels causés par la façon dont l'enquête est construite, par l'attrition et par diverses formes de données manquantes, nous avons utilisé les poids d'échantillonnage fournis par Statistique Canada dans l'ENSP. Comme notre 
modèle est longitudinal, les mesures sont regroupées selon les personnes, en fonction du temps. Par conséquent, les poids ont été appliqués au niveau 2 du modèle sur une personne. Pour évaluer l'attrition liée au décès, le modèle a été comparé à des modèles concurrents modifiés où des personnes qui sont décédées au cours de l'ENSP ont reçu la valeur de 0 en ce qui concerne l'ESAD. Les résultats n'ont montré aucun changement dans les paramètres estimés du niveau 1 ou 2 . Le biais potentiel causé par la conception de l'enquête a été évalué selon la régression de la variance des résidus à partir des modèles de régression finaux sur les poids d'échantillonnage (Rabe-Hesketh et Skrondal, 2006; Wu, 2010). Nous n'avons constaté aucun lien entre la taille de la variance des résidus et de la probabilité dans l'ENSP. Cette même technique a été utilisée pour s'assurer que les schémas de données manquantes ne sont pas liés à la taille de la variance des résidus.

La validation d'un modèle standard fait suite à la division de l'ensemble de données en deux groupes: un groupe de données exploratoires et un groupe de données de confirmation. Dans ce processus, les questions de recherche sont d'abord examinées avec les données exploratoires puis avec les données de confirmation (Fox, 2008; Good et Hardin, 2006). Les résultats présentés ont été obtenus à la suite d'un processus en trois étapes servant à mettre à l'épreuve nos questions de recherche. L'ensemble des données pour les huit cycles a été converti en format long, puis échantillonné de façon aléatoire, sans remplacement, afin d'obtenir deux ensembles de données égaux. Ensuite, l'ensemble de données exploratoires a de nouveau été échantillonné au hasard afin d'obtenir un échantillon de 10 \% (de la quantité totale de l'ensemble de données) à partir duquel la procédure de modélisation a été exécutée. Toutes les variables proposées en théorie ont d'abord été intégrées au modèle de régression. De façon progressive, une approche d'élimination en commençant par la fin a été utilisée, permettant d'éliminer une variable à la fois dans le but de renforcer le modèle. À chaque étape, le modèle a été évalué à l'aide du critère d'Akaike des informations (CAI), du critère bayésien des informations (CBI), des analyses du rapport de vraisemblance (RV) et des diagnostics de régression afin de constater l'amélioration de l'ajustement par rapport à la perte d'ajustement ou d'information. Les modèles exploratoires ont été créés à partir de l'échantillon de $10 \%$, affinés au moyen l'ensemble de données exploratoires, puis testés avec l'ensemble de données de confirmation. Cette approche permet un renforcement majeur de la validité des résultats par une évaluation minutieuse et progressive et par une validation du modèle à partir d'un ensemble de données connexes, mais indépendantes.

\section{RÉSULTATS}

La modélisation statistique de l'ESAD a été réalisée à l'aide d'une série de modèles de régression (voir les tableaux 2 à 5) pour une interprétation à l'échelle individuelle, mais aussi à l'échelle de la population. Dans notre cas, nous mettons l'accent sur les résultats à l'échelle de la population. La partie niveau 2 des modèles statistiques est utilisée pour examiner la variance et la covariance seulement. Nous examinons 
d'abord les résultats pour toutes les cohortes dans les données de l'ENSP avant de porter un regard sur nos deux cohortes analysées en particulier.

\section{ÉTAT DE SANTÉ AUTODÉCLARÉ}

Nous avons élaboré un modèle fiable pour l'ESAD en nous appuyant sur l'échantillon de $10 \%$ et sur la moitié de l'ensemble des données exploratoires de l'ENSP. Nous l'avons ensuite validé en utilisant la moitié de l'ensemble des données de confirmation de l'ENSP, pour un total de 8187 personnes qui ont été modélisées pendant plus de 14 ans, ce qui a permis de générer 42021 observations, avec un nombre moyen d'observations par personne supérieur à 5 . Il s'agit d'une quantité importante de données complètes ou presque complètes au cours des huit cycles de l'ENSP à l'étude. Le modèle permet d'estimer les paramètres de niveau 1, comme les effets fixes, à l'exception du temps et de l'interception. Ces effets fixes peuvent être interprétés comme l'effet prévu ou l'effet moyen de la covariable sur une personne donnée de la population (tous les Canadiens) à partir de laquelle ils ont été tirés. Les estimations du modèle sont présentées au tableau 2.

La valeur de chaque coefficient est interprétée en tenant compte de toutes les autres variables. Le coefficient de Gini est à la fois une interaction avec le temps (trajectoire au fil du temps) et un effet principal.

\section{Temps}

La covariable du temps dans ce modèle à plusieurs niveaux constituait une variable explicative de niveau 1, non significative de l'état de santé autodéclaré. L'effet aléatoire de temps a été intégré sans cette variable explicative. Par conséquent, le modèle a un effet moyen de temps de zéro compte tenu du fait que les estimations des effets aléatoires en fonction de la personne et du temps au niveau 2 doivent avoir obligatoirement une moyenne de zéro également (Snijders et Bosker, 2012). À partir de l'équation (3) nous avons établi que $\beta_{1} \beta_{1}=0$, en raison de son manque de signification statistique. L'effet aléatoire du temps est très significatif avec une statistique $\mathrm{T}$ beaucoup plus élevée que 7 ( $\mathrm{T}$ [pente] = 0,007 / 0,001 = 7, cependant, nous avons arrondi l'erreur type à partir d'une valeur qui, autrement, aurait été arrondie à zéro). La variance globale des pentes reste faible. L'interception comporte toutefois une grande variance avec une statistique $\mathrm{T}$ d'environ 28,4 , ce qui indique une variation importante entre les observations individuelles. 


\section{Tableau 2: Les coefficients de régression ainsi que la variance et la covariance des effets aléatoires pour l'EDSA}

\begin{tabular}{lcccc}
\multicolumn{1}{c}{ Variable dépendante } & État de santé autodéclaré & & \\
\hline $\begin{array}{l}\text { Nombre d'observations ou } \\
\text { regroupements }\end{array}$ & Niveau 1 & 42 O21 & Niveau 2 & 8187 \\
\hline Covariables & Coefficient & p. de la valeur & Intervalle de confiance à $95 \%$ \\
\hline Âge & $-0,125$ & $<0,001$ & $-0,143$ & $-0,107$ \\
Âge moyen & 0,114 & $<0,001$ & 0,096 & 0,133 \\
Personne inactive & $-0,129$ & $<0,001$ & $-0,164$ & $-0,095$ \\
Femme & $-0,060$ & 0,009 & $-0,105$ & $-0,015$ \\
Ratio du revenu du ménage & 0,042 & $<, 001$ & 0,029 & 0,056 \\
Scolarisation & 0,025 & $<0,001$ & 0,019 & 0,030 \\
Coefficient de Gini & 0,616 & 0,451 & $-0,986$ & 2,219 \\
Âge en fonction du temps & $-0,001^{*}$ & 0,039 & $-0,001^{*}$ & 0,000 \\
Ratio du revenu du ménage en fonction & $-0,001$ & 0,416 & $-0,004$ & 0,002 \\
du temps & & & $0,0,371$ & 0,571 \\
Coefficient de Gini en fonction du temps & 0,471 & $<0,001$ & $0,001^{*}$ & 0,000 \\
Femme selon l'âge, en fonction du temps & $0,001^{*}$ & 0,005 & 1,380 & 2,705 \\
Interception (niveau 1) & 2,043 & $<0,001$ & & \\
\hline
\end{tabular}

\begin{tabular}{lcc}
$\begin{array}{l}\text { Variances et covariances des effets } \\
\text { aléatoires }\end{array}$ & Estimation & Erreur type \\
\hline Interception & 0,426 & 0,015 \\
Pente & 0,007 & $0,001^{*}$ \\
Interception selon la pente & $-0,021$ & 0,002 \\
\hline
\end{tabular}

Remarque: Lorsque des estimations sont inférieures aux trois chiffres significatifs autorisés par Statistique Canada, un astérisque est inscrit pour indiquer un arrondissement différent de zéro.

\section{Variables explicatives des statuts démographiques et socio-économiques}

Les femmes enregistrent une différence moyenne de moins 0,06 unité que les hommes sur l'échelle de l'ESAD. Les interactions à deux critères, en fonction du sexe et du temps et d'autres variables qui n'étaient pas significatives, indiquant la trajectoire prévue des hommes et des femmes dans l'étude, pourraient être considérées équivalentes. Une variable d'interaction à trois critères s'est avérée significative et son interprétation par rapport aux variables de l'âge est examinée ci-après. Comme le montre la figure 1, nous observons sans surprise que la trajectoire prévue de l'ESAD en fonction de l'âge décline, mais pas de façon très nette.

La croissance prévue de l'état de santé autodéclaré selon le ratio du revenu d'un ménage était de 0, 042 unité pour chaque niveau de revenu du ménage qui dépasse son seuil de faible revenu relatif (SFR). L'interaction entre le ratio du revenu du ménage et le temps n'était pas considérable sur le plan statistique, mais ce lien a été conservé 
dans le modèle, car le pouvoir explicatif de ce dernier n'aurait pas davantage été amélioré $^{4}$ par le retrait de ce lien. Ainsi, le ratio du revenu du ménage en tant que tel n'a pas eu de répercussions importantes sur la santé durant le parcours de vie des personnes de l'ENSP.

\section{Figure 1: Trajectoire prévue de l'ESAD durant le parcours de vie}

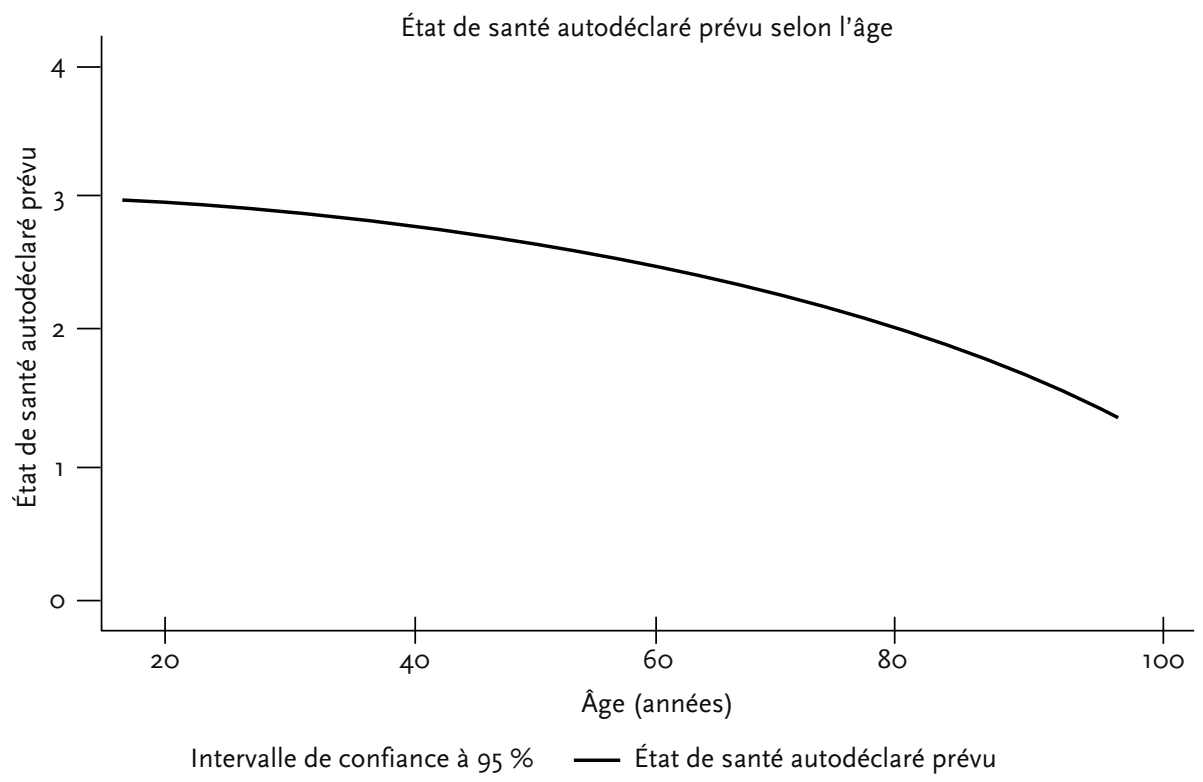

Le lien entre la population active et l'ESAD est très important. Les personnes inactives enregistrent en moyenne une différence de -0,129 unité par rapport aux personnes sur le marché du travail. Bien sûr, il pourrait s'agir d'une causalité inversée, car forcément, les gens en mauvaise santé peuvent être obligés de quitter le marché du travail. Aucun lien statistiquement significatif entre le chômage et l'ESAD n'a été observé, ce qui est quelque peu surprenant compte tenu des conclusions précédentes concernant les conséquences négatives du chômage sur la santé. Une grande partie de cette recherche visait toutefois à comparer des chômeurs et des employés à un moment précis dans le temps. Les conséquences du chômage sur la santé, s’il est de courte durée, peuvent être négligeables au cours d'une vie. Cependant, les conditions d'interaction liées au fait de ne pas faire partie de la population active ou d'être au chômage en fonction du temps ne sont également pas significatives et n'ont pas été intégrées au modèle.

4. Selon le CAI, le CBI, les analyses du rapport de vraisemblance et les diagnostics de régression. 


\section{L'inégalité du revenu}

Nous avons observé que le coefficient de Gini, en tant que mesure de l'inégalité du revenu, avait un lien étrange avec l'ESAD. L'effet sur la santé de ce coefficient seul ne s'est pas avéré statistiquement significatif, mais son interaction avec le facteur temps l'était toutefois. En effet, le coefficient de Gini en fonction du temps peut indiquer une augmentation considérable des ESAD «très bons » ou «excellents», même en présence d'inégalités croissantes (ce qui peut être considéré comme une augmentation à partir de zéro, voir le tableau 3). Bien que le coefficient Gini utilisé seul puisse avoir un léger effet positif ou négatif au fil du temps, cet effet est neutralisé par la tendance apparente des personnes à déclarer un meilleur état de santé avec le temps. Cela pourrait s'expliquer du fait que leur auto-évaluation relative est comparée à celles d'autres personnes nées à la même époque. La relation linéaire observée dans notre analyse a une pente moyenne prévue de 0, 203 unité sur l'échelle de l'état de santé autodéclaré entre des points temporels successifs.

\section{Variables liées à l'âge}

L'âge et les processus associés à l'âge sont fortement liés à l'ESAD. L'effet combiné de l'âge et de l'âge moyen fournit une trajectoire moyenne de la santé des personnes qui ont atteint leur âge moyen dans l'ENSP. Cette pente représente le coefficient pour l'âge et est estimée à -0,125 unité seulement. De plus, elle repose sur l'interaction entre l'âge et le temps. Les changements observés dans les ESAD sont les plus faibles chez les jeunes et s'accentuent avec l'âge. Le taux d'augmentation de la pente est proportionnel à l'interaction entre l'âge et le temps dont l'effet est estimé à -0,001. Ces pentes inégales montrent encore une fois les différences observées entre les premiers baby-boomers et les pré-baby-boomers.

\section{Comparaison entre les premiers baby-boomers et les pré-baby-boomers}

Nous allons maintenant nous concentrer sur nos deux cohortes analysées. Les différences prévues entre la façon dont les premiers baby-boomers et les pré-baby-boomers évaluent leur état de santé sont liées aux différences prévues dans l'interaction de l'âge avec le temps. Comme mentionnée précédemment, aucune valeur exacte n'est fournie, compte tenu des lignes directrices sur la divulgation des renseignements de Statistique Canada. Cependant, la nature de la différence entre les deux groupes est illustrée à la figure 2 ci-dessous et peut être expliquée au moyen d'un exemple. Le tableau 4 montre également les pointages moyens prévus pour chaque groupe, compte tenu de notre modèle et répartis en fonction du temps dans les huit cycles de l'ENSP. 
Tableau 3: L'effet net de l'inégalité du revenu au fil du temps sur l'ESAD

\begin{tabular}{cccc}
\multicolumn{2}{c}{ Variable dépendante } & \multicolumn{2}{c}{ État de santé autodéclaré } \\
\hline \multirow{2}{*}{ Temps } & Coefficient de Gini & $\begin{array}{c}\text { Coefficient de Gini en fonction } \\
\text { du temps }\end{array}$ & Effet net \\
\hline 0 & $0-$ non significatif & $(0,471 \times 0,403) \times 0$ & 0 \\
1 & 0 & $(0,471 \times 0,416) \times 1$ & 0,196 \\
2 & 0 & $(0,471 \times 0,427) \times 2$ & 0,402 \\
3 & 0 & $(0,471 \times 0,430) \times 3$ & 0,608 \\
4 & 0 & $(0,471 \times 0,427) \times 4$ & 0,804 \\
5 & 0 & $(0,471 \times 0,430) \times 5$ & 1,013 \\
6 & 0 & $(0,471 \times 0,429) \times 6$ & 1,212 \\
7 & 0 & $(0,471 \times 0,431) \times 7$ & 1,421 \\
\hline
\end{tabular}

Figure 2: Trajectoires de l'ESAD pour les pré-baby-boomers et les premiers baby-boomers par rapport à l'échelle de temps de l'ENSP



En examinant la différence prévue de 15 ans entre nos cohortes analysées et notre estimation arrondie des paramètres, nous constatons que la cohorte des premiers baby-boomers a atteint la force de l'âge en meilleure santé que celle des pré-babyboomers. Cette estimation a été établie à partir de l'ESAD prévu pour des personnes du même âge à différentes périodes de temps de 15 ans. L'interaction prévue entre l'âge et le temps était de $-0,001$ * et la différence de temps serait de 7,5 unités dans le modèle, ou de 15 ans. Ces résultats laissent supposer que la cohorte des premiers babyboomers se situerait à 0,008 unité plus élevée sur l'échelle de l'ESAD, lorsque l'âge est maintenu constant. La possibilité qu'il s'agisse d'une conclusion aléatoire est margi- 
nale puisque la statistique T obtenue pour ce paramètre n'était que modérée $(-2,07$ avec $\mathrm{p}<0,039)$, ce qui correspond à d'autres résultats de recherche et peut varier en fonction des expériences du parcours de vie. Même s'ils ont atteint l'âge adulte à une époque où les emplois abondaient et où le coût de la vie était relativement bas, les pré-baby-boomers sont nés dans la foulée de la grande dépression et ont peut-être commencé à fumer à un jeune âge. Ils étaient des enfants âgés d'environ 9 à 12 ans au moment de la Seconde Guerre mondiale et étaient susceptibles d'être influencés par les contraintes de l'époque et le culte de la cigarette. Les premiers baby-boomers, bien que confrontés en tant que jeunes adultes à un marché très compétitif de l'emploi, peuvent avoir été plus attentifs à la prévention des maladies, bien que les recherches de Wister (2005) révèlent qu'ils sont plus sujets à l'embonpoint.

Par ailleurs, l'interaction entre l'âge et le temps pour les femmes (l'interaction à trois critères) a également été jugée comme statistiquement significative. La direction prévue de l'effet était à l'opposé de celle de l'interaction générale de l'âge en fonction du temps. L'estimation arrondie permettrait d'obtenir un effet prévu égal, mais à l'opposé, ce qui annulerait l'ensemble des effets de cohorte pour les femmes. Les valeurs réelles ne sont pas égales et l'effet net de cette variable est de corriger ou de réduire la différence prévue entre les femmes, en fonction de leur âge et selon le temps. Cela laisse supposer que la différence entre la façon dont les hommes et les femmes déclarent leur état de santé se modifie avec le temps, les hommes déclarant toutefois une santé moins bonne que les femmes tout au long parcours de vie. Cette valeur était moins susceptible d'être observée par hasard que l'interaction à deux critères de l'âge en fonction du temps, car la statistique $\mathrm{T}$ obtenue pour ce paramètre était de taille modérée à élevée $(2,80$ avec $\mathrm{p}<0,005)$.

\section{Tableau 4: ESAD des premiers baby-boomers et des pré-baby-boomers et différence en pourcentage des cohortes en fonction du temps}

\begin{tabular}{cccc} 
Variable dépendante & État de santé autodéclaré \\
\hline Temps & $\begin{array}{c}\text { Premiers baby- } \\
\text { boomers }\end{array}$ & Pré-baby-boomers & Différence en \% \\
\hline 0 & 2,796 & 2,337 & 20 \\
1 & 2,788 & 2,384 & 17 \\
2 & 2,766 & 2,395 & 15 \\
3 & 2,674 & 2,311 & 16 \\
4 & 2,550 & 2,209 & 15 \\
5 & 2,521 & 2,179 & 16 \\
6 & 2,501 & 2,160 & 16 \\
7 & 2,539 & 2,168 & 17 \\
\hline
\end{tabular}

Le modèle de régression pour l'ESAD a été appliqué aux premiers baby-boomers et aux pré-baby-boomers sélectionnés dans l'ensemble complet des données. Nous avons donc créé deux sous-échantillons composés de 395 et de 648 personnes pour un nombre total d'observations de 2165 et de 3885 respectivement, et un nombre moyen 
d'observations par personne durant les 8 cycles de 5,5 et de 6, respectivement. Une analyse presque hiérarchique des modèles a été réalisée et les résultats se trouvent dans le tableau $5^{5}$.

Une série de conclusions se dégagent pour les deux cohortes. Par exemple, les plus grandes différences entre les coefficients de ces cohortes concernent les facteurs socioéconomiques. En effet, la cohorte des premiers baby-boomers présente un avantage beaucoup plus important qui se traduit par une augmentation du ratio du revenu du ménage. Les faibles ratios de revenu du ménage sont plus étroitement liés à une moins bonne santé pour la cohorte des premiers baby-boomers que pour celle des pré-babyboomers.

Tableau 5: Les coefficients de régression pour les premiers baby-boomers, les prébaby-boomers et la proportion relative (coefficient des premiers babyboomers divisé par celui des pré-baby-boomers) entre les cohortes en fonction des variables explicatives de SSE, des variables explicatives de SSE et du sexe, et des nombreux modèles variables

\begin{tabular}{|c|c|c|c|c|c|c|c|}
\hline & Variable dépendante & & & État d & anté autodéclaré & & \\
\hline & $\begin{array}{l}\text { ombre d'observations ou } \\
\text { regroupement: }\end{array}$ & $\begin{array}{c}\text { Premiers } \\
\text { baby- } \\
\text { boomers }\end{array}$ & Niveau 1 & 3885 & $\begin{array}{l}\text { Pré-baby- } \\
\text { boomers }\end{array}$ & Niveau 1 & 2165 \\
\hline & & & Niveau 2 & 648 & & Niveau 2 & 395 \\
\hline Bloc & Covariables & & $\begin{array}{l}\text { Premiers baby- } \\
\text { boomers }\end{array}$ & & Pré-baby-boomers & Proportion re & lative \\
\hline 1 & Âge & & $-0,083$ & & $-0,081$ & 1,02 & \\
\hline & Âge moyen & & 0,067 & & 0,113 & 0,59 & \\
\hline & Personne inactive & & $-0,189$ & & $-0,251$ & 0,75 & \\
\hline & Femme & & - & & - & - & \\
\hline & $\begin{array}{l}\text { Ratio du revenu du } \\
\text { ménage }\end{array}$ & & 0,070 & & 0,009 & 7,78 & \\
\hline & Scolarisation & & 0,026 & & 0,036 & 0,72 & \\
\hline & Coefficient de Gini & & - & & - & - & \\
\hline & $\begin{array}{l}\text { Ratio du revenu du mén } \\
\text { le temps }\end{array}$ & age selon & $-0,008$ & & 0,011 & $-0,73$ & \\
\hline & Âge selon le temps & & 0,002 & & 0,001 & 2,00 & \\
\hline & $\begin{array}{l}\text { Coefficient de Gini } \\
\text { selon le temps }\end{array}$ & & - & & - & - & \\
\hline & $\begin{array}{l}\text { Femme selon l'âge, } \\
\text { selon le temps }\end{array}$ & & - & & - & - & \\
\hline & Interception (niveau 1) & & 2,785 & & $-0,239$ & $-11,65$ & \\
\hline 2 & Âge & & $-0,083$ & & $-0,074$ & 1,12 & \\
\hline & Âge moyen & & 0,067 & & 0,105 & 0,64 & \\
\hline & Personne inactive & & $-0,186$ & & $-0,266$ & 0,70 & \\
\hline
\end{tabular}

5. Une fois de plus, toutes les estimations de paramètres pour les sous-échantillons se situaient dans les intervalles de confiance de l'ensemble des données du modèle. Le chevauchement des intervalles de confiance a montré que les estimations obtenues à partir des sous-échantillons n'étaient pas plus différentes de celles de l'ensemble complet des données que ce qui pourrait être considéré comme dû au hasard. 


\begin{tabular}{|c|c|c|c|c|}
\hline & Femme & $-0,082$ & 0,214 & $-0,38$ \\
\hline & $\begin{array}{l}\text { Ratio du revenu du } \\
\text { ménage }\end{array}$ & 0,068 & 0,013 & 5,23 \\
\hline & Scolarisation & 0,025 & 0,04 & 0,63 \\
\hline & Coefficient de Gini & - & - & - \\
\hline & $\begin{array}{l}\text { Ratio du revenu du ménage en } \\
\text { fonction du temps }\end{array}$ & $-0,007$ & 0,01 & $-0,70$ \\
\hline & $\begin{array}{l}\text { Âge en fonction du } \\
\text { temps }\end{array}$ & 0,002 & 0,001 & 2,00 \\
\hline & $\begin{array}{l}\text { Coefficient Gini en } \\
\text { fonction du temps }\end{array}$ & - & - & - \\
\hline & $\begin{array}{l}\text { Femme selon l'âge, } \\
\text { selon le temps }\end{array}$ & O & ० & - \\
\hline & Interception (niveau 1) & 2,869 & $-0,324$ & $-8,85$ \\
\hline 3 & Âge & $-0,081$ & $-0,112$ & 0,72 \\
\hline & Âge moyen & 0,047 & 0,117 & 0,40 \\
\hline & Personne inactive & $-0,197$ & $-0,276$ & 0,71 \\
\hline & Femme & $-0,081$ & 0,214 & $-0,38$ \\
\hline & $\begin{array}{l}\text { Ratio du revenu du } \\
\text { ménage }\end{array}$ & 0,069 & 0,019 & 3,63 \\
\hline & Scolarisation & 0,025 & 0,038 & 0,66 \\
\hline & Coefficient de Gini & 13,679 & 13,013 & 1,05 \\
\hline & $\begin{array}{l}\text { Ratio du revenu du ménage, selon } \\
\text { le temps }\end{array}$ & 0,010 & 0,005 & 2,00 \\
\hline & $\begin{array}{l}\text { Âge en fonction du } \\
\text { temps }\end{array}$ & $-0,007$ & 0,009 & $-0,78$ \\
\hline & $\begin{array}{l}\text { Coefficient Gini selon } \\
\text { le temps }\end{array}$ & $-1,236$ & $-0,586$ & 2,11 \\
\hline & $\begin{array}{l}\text { Femme selon l'âge, } \\
\text { selon le temps }\end{array}$ & 0,001 & ০,০০০ & - \\
\hline & Interception (niveau 1) & $-1,788$ & $-4,123$ & 0,43 \\
\hline
\end{tabular}

Même si le coefficient de Gini en fonction du temps pour les deux cohortes de l'échantillon est négatif, l'effet pour la cohorte des premiers baby-boomers est le double de celui de la cohorte des pré-baby-boomers et est statistiquement significatif avec une statistique $T$ importante de $-3,05(\mathrm{p}<0,002)$. Le fait que l'effet d'interaction du coefficient de Gini avec le temps l'emporte sur l'effet principal de ce coefficient suggère qu'en fonction du temps et des parcours de vie, au fur et à mesure que le coefficient de Gini passe de 0 à une unité supérieure, ce qui indique un plus grand degré d'inégalité, une situation est préjudiciable à la santé des personnes. Les valeurs semblent imposantes, car les infimes changements dans le coefficient de Gini luimême ont seulement rendu visibles d'autres effets observés dans le modèle. Par exemple, les coefficients de l'âge et des interactions avec l'âge ont la même importance. Seul l'effet de l'interaction du coefficient de Gini avec le temps se démarque, car il suggère que même d'infimes changements dans l'inégalité du revenu au fil du temps ont des effets percutants sur la santé.

Les conditions nécessaires à une transition en santé du milieu à la fin de la vie pour les premiers baby-boomers et les pré-baby-boomers ne sont pas les mêmes. Le 
statut socio-économique et les inégalités du revenu comptent, mais davantage pour les premiers baby-boomers que pour les pré-baby-boomers.

\section{RÉSUMÉ ET CONCLUSIONS}

Cette étude a examiné les relations entre le statut socio-économique, les inégalités du revenu et les différences entre les deux cohortes analysées en particulier, celle des premiers baby-boomers et celle des pré-baby-boomers au Canada, en ce qui concerne l'ESAD durant le passage entre le milieu et la dernière partie de la vie.

Les liens entre les inégalités et la santé tels qu'évalués à partir de l'ESAD sont très complexes. Quelques facteurs peuvent expliquer ce phénomène. Tout d'abord, le modèle de régression pour l'ESAD a été fondé sur une grande partie du parcours de vie, ce qui peut produire un effet moyen ou fixe des coefficients de Gini difficile à déterminer compte tenu de sa différence de fonctionnement tout au long de cette période. Les différences entre les cohortes par rapport au coefficient de Gini suggèrent que l'effet de ce coefficient est plus important pour la cohorte des premiers baby-boomers que pour celle des pré-baby-boomers. Par conséquent, nous pouvons présumer que les parcours de vie peuvent être parsemés de périodes critiques où la santé est davantage touchée par les inégalités du revenu, même si les effets globaux sont plus linéaires.

Ensuite, la façon dont les inégalités influencent l'ESAD peut aussi varier. Les différences entre les cohortes analysées indiquent la possibilité que les inégalités du revenu aient eu moins de répercussions sur l'ESAD dans le passé qu'elles en ont aujourd'hui. En effet, par le passé, les gens étaient peut-être moins conscients du fait que leur qualité de vie ou de leur santé était liée au salaire qu'ils gagnaient, ou moins conscients de leur situation par rapport aux autres en matière de revenu. L'accès aux médias et ce qui est connu comme un comportement de recherche d'ascension sociale peuvent expliquer ces changements au fil du temps. La théorie de Levine, Frank et Dijk (2010) prévoit que l'augmentation des dépenses des titulaires de revenus supérieurs et la visibilité accrue de ces dépenses incitent les personnes qui se situent juste en dessous d'eux sur l'échelle des revenus à dépenser davantage, puis le groupe suivant, et ainsi de suite, ce qui peut augmenter la sensibilité aux inégalités du revenu et de leur croissance.

Dans l'ensemble, les avantages d'avoir un bon revenu par rapport aux autres, d'être sur le marché du travail ou d'être employé et d'avoir un bon niveau de scolarisation sont encore des déterminants très importants de la santé et de la qualité de vie liée à la santé durant le parcours de vie. Il s'agit d'une constatation uniforme parmi les mesures de santé et pour les deux cohortes que nous avons analysées. Cependant, il semble que les facteurs socio-économiques ne représentent qu'un aspect important parmi tant d'autres. De fortes augmentations d'inégalités du revenu peuvent avoir des conséquences importantes et directes pour la transition des cohortes du milieu à la fin de la vie. Cette recherche nous a permis de déterminer que l'ESAD est étroitement lié au statut socio-économique et que l'accroissement des inégalités au fil du temps a des répercussions en particulier sur le bien-être des premiers baby-boomers. Toutefois, ce 
que nous avons pu constater concernant la transition du milieu à la fin de la vie, tant sur le plan social qu'économique, pourrait ne plus s'appliquer aux futures cohortes et même aux derniers baby-boomers.

\section{BIBLIOGRAPHIE}

Avison, W. R., J. Ali and D. Walters (2007), «Family Structure, Stress, and Psychological Distress: A Demonstration of the Impact of Differential Exposure», Journal of Health and Social Behavior, $\mathrm{n}^{\circ} 48$, p. 301-317.

Banks, J., M. M. Маяmot, Z. Oldfield and J. Smith (2006), «Disease and Disadvantage in the United States and in England», Journal of the American Medical Association, n 295, p. 2037-2045.

Black Report (1980), Inequalities in Health: Report of a Research Working Group, London, Department of Health and Social Security.

Сотt, C., M. Gignac and E. Badley (1999), «Determinants of Self-Rated Health for Canadians with Chronic Disease and Disability", Journal of Epidemiology and Community Health, $\mathrm{n}^{\circ}$ 53, p.731-736.

Duncan, G. J., M. C. Daly, P. McDonough and D. R. Williams (2002), «Optimal Indicators of Socioeconomic Status for Health Research ", American Journal of Public Health, 92 (7), p.1151-1157.

EnG, K. and D. Feeny (2007), «Comparing the Health of Low Income and Less Well Educated Groups in the United States and Canada», Population Health Metrics, 5(10), retrieved from www. pophealthmetrics.com/content $/ 5 / 1 / 10$

Feeny, D., Kaplan, M. S., Huquet, N. and B. H. McFarland (2010), «Comparing population health in the United States and Canada», Population Health Metrics, 8(8), retrieved from www.pophealthmetrics. com/content $/ 8 / 1 / 18$

Feeny, D., W. Furlong, G. W. Torrance et al. (2002), Multiattribute and Single-Attribute Utility Functions for the Health Utilities Index Mark 3 System, Medical Care, 40 (2), p. 113-128.

Ferraro, K. F. and T. P. Shippee (2009), «Aging and Cumulative Inequality: How Does Inequality Get Under the Skin?», The Gerontologist, 49 (3), p. 333-43.

Fox, J. (2008), Applied Regression Analysis and Generalized Linear Models, Second Edition, Thousand Oaks, CA, Sage Publications, Inc.

Frenette, M., D. Green and K. Milligan (2006), «Revisiting Recent Trends in Canadian After-Tax Income Inequality Using Census Data», Statistics Canada Business and Labour Market Analysis Paper, Catalogue 11F0019, $\mathrm{n}^{\circ}$ 274, Statistics Canada, Ottawa.

Gelman, A. and J. Hall (2007), Data Analysis Using Regression and Multilevel/Hierarchical Models, Cambridge, Cambridge University Press.

Good, P. I. and J.W. Hardin (2006), Common Errors in Statistics and How to Avoid Them, Hoboken, NJ, John Wiley \& Sons, Inc.

Heisz, A. (2007), «Income Inequality and Redistribution in Canada: 1976 to 2004», Statistics Canada Analytical Paper, Catalogue n ${ }^{\circ} 11$ F0019MIE - no 298.

Hertzman, C. and S. Arjumand (2009), "Population Health and the Dynamics of Collective Development», in P. A. Hall and M. Lamont (dir.), Successful Societies: How Institutions and Culture Affect Health, Cambridge, Cambridge University Press, p. 23-52.

Horsman, J.R. (2012), Health Utilities Inc. Health Related Quality-of-Life, retrieved from www. healthutilities.com

House, J. (2002), «Understanding Social Factors and Inequalities in Health: $20^{\text {th }}$ Century Progress and $21^{\text {st }}$ Century Prospects", Health and Social Behavior, 43, p. 125-142.

Huquet, N., M.S. Kaplan and D. Feeny (2007), «Socioeconomic Status and Health-Related Quality of Life Among Elderly People: Results from the Joint Canada/United States Survey of Health, Social Science and Medicine, 66(4), p. 803-810.

Idler, E. and Y. Benyamini (1997), «Self-Rated Health and Mortality: A Review of Twenty-Seven Community Studies», Journal of Health and Social Behavior, 38, p. 21-27. 
Idler, E., L. Russell and D. Davis (1992), «Survival, Functional Limitations, and Self-Rated Health in the NHANES I Epidemiologic Follow-Up Study», American Journal of Epidemiology, 152, p. 874-883.

Kreyszig, E. (1999), Advanced Engineering Mathematics, New York, John Wiley \& Sons.

Levine, A., R. Frank and O. Dijk (2010), «Expenditure Cascades», available at www.ssrn.com/abstract $=1690612$ or http $: / /$ dx.doi.org/10.2139/ssrn.1690612.

Little, R. J. A. and D. B. Rubin (2002), Statistical Analysis with Missing Data (2 ${ }^{\text {nd }}$ ed.). Hoboken, NJ, John Wiley \& Sons, Inc.

Maio, F. D.G. and E. Kemp (2010), «The Deterioration of Health Status Among Immigrants to Canada», Global Public Health, 5 (5), p. 462-478.

Mansson, N. and L. Rastam (2001), "Self-Rated Health as a Predictor of Disability, Pension and Death - A Prospective Study of Middle-Aged Men», Scandinavian Journal of Public Health, 29, p.151-158.

McDaniel, S. A. (2011), "Understanding Health and Illness Behaviour Sociologically», Sociopedia, publication of the International Sociological Association, July 2011, www.sagepub.net/isa/admin/ viewPDF.aspx? \&art=Health \%20and \%20Illness.pdf

McDaniel, S. A. and P. Bernard (2011), «Life Course as a Policy Lens», Canadian Public Policy, 37 (1), S1-S21.

McDaniel, S. A., A. Gazso and S. Um (2013), «Generationing Relations in Challenging Times: Americans and Canadians in Mid-Life in the Great Recession », Current Sociology, forthcoming.

McDonough, P., D. Worts and A. Sacker (2009), «Welfare Regimes and Social Inequalities in Health Dynamics: A Comparative Analysis of Panel Data from Britain, Denmark, Germany and the U.S.», Paper presented at the $7^{\text {th }}$ Canadian Research Data Centres Network Conference, Health Over the Life Course, 15-16, October, London, Ontario.

McGrail, K. M., E. van Doorslaer, N. A. Ross and C. Sammartin (2009), «Income-Related Health Inequalities in Canada and the United States: A Decomposition Analysis ", American Journal of Public Health, 99 (10), p.1856-1863.

Ménard, F. P., C. Le Bourdais et D. Hamplové (2010), «Les parcours familiaux des Canadiens âgés d'aujourd'hui et de demain: Aperçu des changements démographiques des 40 dernières années", dans Relations intergénérationnelles. Enjeux démographiques, Actes du Colloque international de Genève de l'Association internationale des démographes de langue française (AIDELF), 14 p.

Monette, G. (2010), Longitudinal Data Analysis with Mixed Models, York University Summer Program in Data Analysis 2010, retrieved from www.math.yorku.ca/people/georges/Files/Longitudinal_Data_ Analysis.pdf

Morin, A. J. S., C. Maïano, H.W. Marsh, M. Janosz and B. Nagengast (2011), "The Longitudinal Interplay of Adolescents' Self-Esteem and Body Image: A Conditional Autoregressive Latent Trajectory Analysis», Multivariate Behavioral Research, 46 (2), p.157-201.

Mossey, J. and E. Shapiro (1982), "Self-Rated Health: A Predictor of Mortality Among the Elderly», American Journal of Public Health, 72, p. 800-808.

PARK, J. (2011), « Retirement, Health and Employment among those 55 plus», Perspectives on Labour and Income, Statistics Canada, Catalogue n ${ }^{\circ} 75-001-\mathrm{X}, 2011$, p. 3-12.

Picot, G. and J. Myles. (2005), «Income Inequality and Low Income in Canada: An International Perspective», Statistics Canada, Analytical Paper, Catalogue n 11F0019MIE, n² 240.

Pinquart, M. (2001), "Correlates of Subjective Health in Older Adults: A Metaanalysis », Psychology and Aging, 16, p. 414-426.

Prus, S. G. (2011), «Comparing Social Determinants of Self-Rated Health Across the United States and Canada ", Social Science and Medicine, 73, p. 50-59.

Rabe-Hesketh, S. and A. Skrondal, (2006), Multilevel Modelling of Complex Survey Data, Journal of the Royal Statistical Society A, 169, Part 4, p. 805-827.

Ross, N. A., R. Garner, J. Bernire, D. H. Feeny, M.S. Kaplan, B. McFarland et al. (2012), «Trajectories of Health-Related Quality of Life by Socio-Economic Status in a Nationally Representative Canadian Cohort», Journal of Epidemiology and Community Health, 66, p. 593-598. 
Sacker, A., R. D. Wiggins, M. Bartley and P. McDonough (2007), «Self-Rated Health Trajectories in the United States and the United Kingdom: A Comparative Study, » American Journal of Public Health, 97 (5), p. 812-818.

Seabrook, J. and W. Avison (2012), «Socioeconomic Status and Cumulative Disadvantage Processes across the Life Course: Implications for Health Outcomes», Canadian Review of Sociology, 49 (1), p. 50-68.

Singh-Manoux, A. J. E. Ferrie, T. Chandola and M. Marmot (2004), «Socioeceonomic Trajectories across the Life Coures and Health Outcomes in Midlife: Evidence for the Accumulation Hypothesis?», International Journal of Epidemiology, 33, p. 1072-1079.

Snijders, T. A. B. and R. J. Bosker (2012), Multilevel Analysis: An Introduction to Basic and Advanced Multilevel Modeling, ( $2^{\text {nd }}$ ed.), Thousand Oaks, CA, Sage Publications Inc.

Statistics Canada (n.d.), Table 202-0705, Gini Coefficients of Market, Total and After-Tax Income, by Economic Family Type, Annual (number), CANSIM (database), version updated April 13, 2011, www5.statcan.gc.ca/cansim/pick-choisir ?lang=eng\&p2=33\&id=2020705

Statistics Canada (2013), «High Income Trends among Canadian Taxfilers, 1982-2010», The Daily, January, 28, 2013.

Turner, R.J., B. Wheaton and D. A. Lloyd (1995), «The Epidemiology of Social Stress», American Sociological Review, 60, p. 104-125.

Van Doorslaer, E. and U. Gerdtham (2003), «Does Inequality in Self-Assessed Health Predict Inequality in Survival by Income? : Evidence from Swedish Data », Social Science and Medicine, 38, p.1621-1629.

White, H. L., F. I. Matheson, R. Moineddin, J. R. Dunn and R. H. Glazier (2011), «Neighbourhood Deprivation and Regional Inequalities in Self-Reported Health Among Canadians: Are We Equally at Risk?", Health and Place,17 (1), p. 361-369.

Wilkins, J. Russell, A. Berthelot and Ng. Edward (2002), «Trends in Mortality by Neighbourhood Income in Urban Canada from 1971 to 1996 », Health Reports, Statistics Canada, 13, p.1-29.

Wilkinson, R.G. (2006), The Impact of Inequality: How to Make Sick Societies Healthier, New York, New Press.

Wilkinson, R.G. and K. E. Pickett (2006), «Income Inequality and Population Health: A Review and Explanation of the Evidence», Social Science and Medicine, 62, p. 1768-1784.

Willson, A. (2009), " "Fundamental Causes" of Health Disparities: A Comparative Analysis of Canada and the United States", International Sociology, 24 (1), p. 93-113.

Wister, A. (2005), Baby Boomer Health Dynamics: How Are We Aging?, Toronto, University of Toronto Press.

Wu, L. (2010), Mixed Effects Models for Complex Data, Boca Raton, FL, CRC Press, Taylor \& Francis Group. 